maximized and Doppler-free spectra of all three energy levels can be obtained.

The authors are indebted to Dr. S. Stenholm for stimulating our interest in this problem.

\section{A7 TWO-PHOTON ABSORPTION MEASUREMENTS WITH ULTRASHORT BROAD BAND LIGHT CONTINUA}

\author{
A. PENZKOFER, W. FALKENSTEIN and W. KAISER \\ Physik-Department der Technischen Universität \\ München, Munich, Fed. Rep. Germany
}

A new technique of two-photon spectroscopy is described. The spectral attenuation of a broad frequency pulse is studied in the presence of a second intense monochromatic pulse. The method has several important advantages: (i) The two-photon absorption spectrum can be measured by a single light pulse. (ii) It is readily possible to distinguish between two-photon absorption and two-step transitions by adjusting the temporal overlap of the two picosecond pulses. (iii) High light intensities can be applied to the samples without material damage.

In our experiments a mode-locked Nd-glass laser was used. Single picosecond light pulses were selected from the early part of the pulse train and amplified to an energy of $\sim 3 \mathrm{~mJ}\left(\Delta t_{\mathrm{L}} \simeq 6 \mathrm{ps}\right.$ fwhm, $\left.\Delta \widetilde{\nu}_{\mathrm{L}} \simeq 3 \mathrm{~cm}^{-1} \mathrm{fwhm}\right)$. The picosecond broad-band light continua were generated by parametric four-photon interaction of the single laser pulse in liquid water $[1]$.

In the new technique of picosecond two-photon spectroscopy, the intense monochromatic laser pulse and the broadband picosecond light continuum pass simultaneously through the two-photon absorbing sample. With optical multi-channel analy sers the two-photon absorption spectrum was measured over a wide frequency range in a single shot.

As an example the nonlinear absorption of CdS single crystals was measured. The two-photon absorption crosssections in the energy range between 2.4 and $3.5 \mathrm{eV}$ were determined. New information is obtained of the two-photon absorption of $\mathrm{CdS}$ on account of the improved accuracy and higher sensitivity of our technique.

As an application of two-photon absorption we have investigated a system where the intensity of ultrashort light pulses is directly determined. The one-to-one correspondence between the energy transmission through a two-photon absorbing medium and the input peak intensity of the laser pulse was utilized to determine calibration curves for intensity determination. Experimentally straightforward energy transmission measurements are made. In a first experiment we used rutile crystals as nonlinear absorbers. The input peak intensity was determined by simultaneously measuring the energy, duration and beam diameter of the light pulses. Twophoton absorption measurements with picosecond ligth con- tinua showed a smooth absorption behavior in the spectral range between 5300 and $6260 \AA$. The obtained calibration curves may be used for intensity detection of the second harmonic of mode-locked Nd-glass laser pulses and of modelocked dye laser pulses.

References

[1] A. Penzkofer, A. Laubereau and W. Kaiser, Phys. Rev. Lett. 31 (1973) 863.

\section{A8 LINESHAPE ANOMALY IN INFRARED- MICROWAVE DOUBLE RESONANCE M. TAKAMI \\ Institute of Physical and Chemical Research, Wako, Saitama 351, Japan}

The infrared-microwave double resonance effect has been observed in $\mathrm{H}_{2} \mathrm{CO}$ with a Zeeman-tuned $3.51 \mu \mathrm{m} \mathrm{He}-\mathrm{Xe}$ laser [1]. The effect of double resonance is observed as a variation of the infrared absorption when the microwave frequency is swept over the rotational transition of $\mathrm{H}_{2} \mathrm{CO}$. The observed pressure dependence of the signal shows that the inversion of the signal occurs at a certain pressure where the anomalous lineshapes are observed. Fig. 1 shows the observed lineshapes with the double resonance cell in the laser cavity. Fig. $1 \mathrm{~b} \mathrm{de-}$

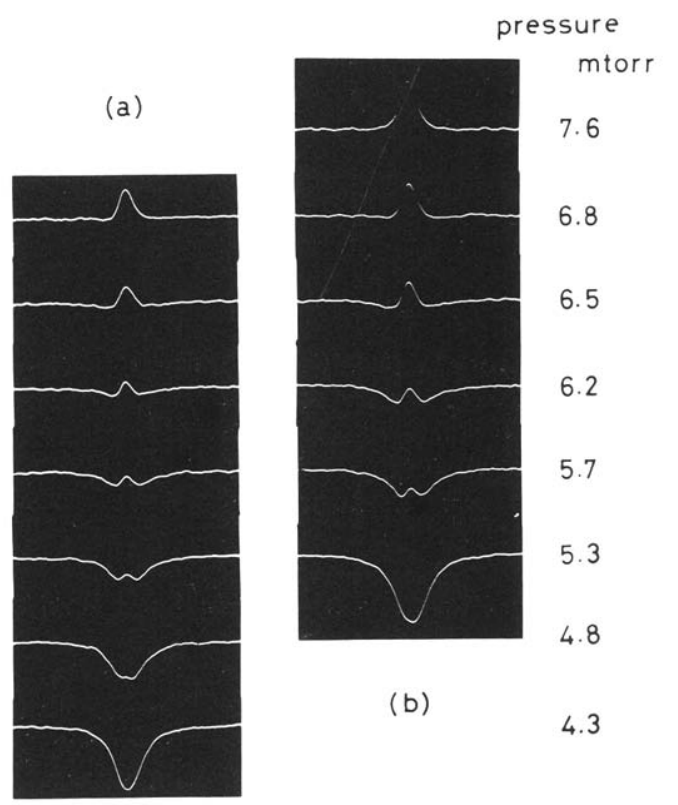

Fig. 1. Observed lineshapes. 